\title{
CRONOLOGÍAS GLACIALES DEL SECTOR NE DEL NEVADO COROPUNA (PERÚ): IMPLICACIONES GEOMORFOLÓGICAS Y PALEOCLIMÁTICAS
}

\author{
José Úbeda \\ Departamento de Análisis Geográfico Regional y Geografía Física. Universidad Complutense de Madrid. \\ joseubeda@ghis.ucm.es
}

\section{RESUMEN}

El trabajo estudia el sector NE del Nevado Coropuna, un complejo de edificos volcánicos que se encuentra en la cordillera occidental de los Andes Centrales del sur de Perú, en el contacto del Altiplano andino con la rampa que desciende hacia la costa del Océano Pacífico. El recuento de isótopos cosmogénicos ${ }^{36} \mathrm{Cl}$ de 8 muestras de superficies de bloques morrénicos indica que el último máximo avance glaciar local sucedió entre 21 y 11 ka, durante el estadio isotópico marino MIS 2. El análisis de las fechas glaciales sugiere nuevas interpretaciones paleoclimáticas y geomorfológicas de gran interés a escala local y regional.

Palabras Clave: Último Máximo Glacial Local, Último Glacial Local, edades de exposición de superficies, bloques morrénicos, plancton de agua dulce, paleotemperatura de la superficie del mar.

\section{ABSTRACT}

This paper studies a glaciated area on this volcanic complex which is located on the limit between the andean Altiplano and the ramp reaching the Pacific Ocean coast. The report of ${ }^{36} \mathrm{Cl}$ cosmogenic isotopes of 8 surface samples on moraine boulders show several local glacier maximum advances among 21 and $11 \mathrm{kyr}$, over Marine Isotope Stage MIS 2. Glacial data analysis suggests very interesting new paleoclimatic and geomorphologic interpretations, both in local and regional scales.

Fecha de recepción: septiembre 2011.

Fecha de aceptación: enero 2013. 
Key words: Local Last Glacial Maximum, Local Late Glacial, surface exposure dating, moraine boulders, freshwater plankton, sea surface paleotemperature.

\section{INTRODUCCIÓN}

Hasta la última década del siglo XX la mayoría de las dataciones de la última máxima expansión de los glaciares de los Andes Centrales se establecieron empleando ${ }^{14} \mathrm{C}$, obteniendo un amplio rango de resultados que fueron recopilados por Clapperton (1993). Esas investigaciones se enfrentaron con la dificultad de encontrar materia orgánica en las elevadas altitudes de las montañas tropicales. En los últimos años las técnicas de datación por exposición de superficies (SED) han permitido deducir nuevas cronologías glaciales que han sido ampliamente discutidas en Smith et al. (2008); Zech et al. (2008) y Bromley et al. (2009). Las evidencias geomorfológicas sugieren que algunas montañas de los Andes han permanecido por encima de la altitud de la línea de equilibrio de los glaciares (Equilibrium Line Altitude, ELA o paleoELA) desde el Plioceno, permitiendo la instalación, conservación y desarrollo de masas de hielo, pero otras no han alcanzado suficiente altitud hasta el Cuaternario reciente (Clapperton, 1993). La interpretación geomorfológica de las SED de los Andes Centrales permite reconocer una fase de expansión glaciar muy prolongada, a escala regional, que se extiende desde antes del Last Glacial Maximum global (LGM) hasta el Late Glacial Global (LG). Además, se observan sincronías y asincronías en la cronología de la expansión glacial inicial, entre valles de una misma región o distintas regiones de los Andes Centrales, así como con respecto al calendario glacial global. Muchas SED glaciales muestran un progresivo retraso hacia el sur y el oeste de los Andes Centrales, en el mismo sentido que se reduce la distancia al área fuente de la corriente de Humboldt y se incrementa la aridez del clima. En la Cordillera Blanca $\left(\sim 8-10^{\circ} S\right)$ Farber et al. (2005) han descrito avances casi contemporáneos a la progresión de los inlandsis de las altas latitudes. Sin embargo, en latitudes más meridionales como la Cordillera Real de Bolivia y la región de Cochabamba $\left(\sim 15-17^{\circ} \mathrm{S}\right.$, Zech et al., 2007; Zech et al., 2008) encontraron una fase de máxima expansión inicial más tardía seguida por entre uno y tres reavances, dependiendo de la altitud de la que procedían las masas de hielo. Las únicas cronologías glaciales de la cordillera occidental publicadas hasta el momento proceden del complejo volcánico Nevado Coropuna y sugieren que la máxima expansión inicial fue todavía más reciente (Bromley et al., 2009). Las cronologías de sus pulsaciones posteriores presentan una buena correlación con las de las demás regiones de la cordillera. En conjunto, las SED del complejo volcánico indican que las fases de avance de los paleoglaciares se prolongaron desde un Local Last Glacial Maximum (LLGM), considerablemente más tardío que el LGM, hasta un Local Late Glacial (LLG), contemporáneo al LG. En adelante se utilizará el acrónimo compuesto LLGM-LLG para hacer referencia a ese amplio periodo de expansión glaciar observado en el Nevado Coropuna.

La intención de este trabajo es contribuir al conocimiento de la evolución del sistema glaciar del complejo volcánico durante el Pleistoceno final. Con ese objetivo se han establecido las SED de bloques de las morrenas depositadas durante el último máximo avance de las masas de hielo, midiendo la acumulación en la superficie de las rocas de ${ }^{36} \mathrm{Cl}$, un isótopo 
cosmogénico que no ha sido empleado con anterioridad en los Andes Centrales. Con el mismo procedimiento se ha datado una colada de lava que por su contexto geomorfológico se había considerado de edad holocena. Su cronología confirma que el Nevado Coropuna es un volcán activo y la necesidad de evaluar el riesgo hidrovolcánico que afecta a la población porque debido a la extrema aridez del clima se concentra entorno a los principales colectores fluviales (Úbeda, 2010).

\section{II. ÁREA DE ESTUDIO}

El Nevado Coropuna (1531S, $\left.72^{\circ} 39^{\prime} \mathrm{W}, 6377 \mathrm{msnm}\right)$ se encuentra en el borde meridional del altiplano, $150 \mathrm{~km}$ al NW de la ciudad de Arequipa (sur de Perú), y forma parte de la divisoria de las cuencas hidrográficas de los ríos Ocoña y Majes, en la vertiente del Pacífico de los Andes Centrales (figura 1). Los edificios volcánicos se han desarrollado sobre un sistema de deslizamientos gigantes que afectan al borde sur del altiplano, cuya serie estratigráfica culmina a techo con gruesas capas de ignimbritas del Plioceno inicial (Caldas, 1993; Núñez \& Valenzuela, 2001; Olschausky \& Dávila, 1994). La evidencia geomorfológica (figura 2) sugiere que la actividad volcánica se detuvo durante la última glaciación y se ha reactivado mucho más recientemente, cuando los frentes de las masas de hielo retrocedieron hasta posiciones próximas a las actuales. Las unidades que reflejan esos procesos se han representado en los perfiles topográficos (figura 3) señalados en el mapa geomorfológico (figura 2).

Durante el Holoceno se registraron tres erupciones que emitieron las cenizas que recubren la rampa proglaciar de la vertiente septentrional, además de tres coladas de lava que se canalizan por el interior de los valles glaciares de las vertientes oeste, noreste y sureste del complejo volcánico. Las coladas de lava sólo están afectadas por las acciones erosivas de las masas de hielo en el sector de cabecera delimitado por las morrenas depositadas en la Little Ice Age (LIA). Por esa razón las coladas de lava se han considerado de época holocena (Úbeda, 2010). El complejo volcánico Nevado Coropuna consiste en un grupo de estratovolcanes coalescentes que superan los $6200 \mathrm{~m}$ de altitud. La cumbre del edifico NW alcanza 6377 msnm y es la cota culminante del complejo volcánico. El área de cumbres está cubierta actualmente por un sistema glaciar compuesto por varias decenas de lenguas que descienden por las vertientes en todas direcciones hasta alcanzar entre 5200 y $5600 \mathrm{msnm}$. Racoviteanu (2004) y Racoviteanu et al. (2007) midieron la superficie total de las masas de hielo reclasificando automáticamente una imagen registrada por el satélite ASTER en octubre de 2000, obteniendo un resultado de $60.8 \mathrm{~km}^{2}$. Sin embargo, trabajos más recientes (Úbeda, 2010) han cartografiado las masas de hielo diferenciándolas con criterios geomorfológicos de las vertientes cubiertas exclusivamente por nieve. En esa tarea se emplearon dos ortofotos de alta resolución y una imagen del satélite ASTER en la que el sistema glaciar aparece sin cubierta nival. El resultado son tres mediciones de la superficie del sistema glaciar, en 1955 $\left(56.1 \mathrm{~km}^{2}\right), 1986\left(54.1 \mathrm{~km}^{2}\right)$ y $2007\left(46.6 \mathrm{~km}^{2}\right)$. La aceleración del proceso de deglaciación que se deduce de la comparación de las dos series temporales (1955-1986 y 1986-2007) es coherente con la tendencia al calentamiento global que describen los informes del Panel Intergubernamental del Cambio Climático (IPCC 2007). En el Nevado Coropuna no hay estaciones meteorológicas, pero en 2007 se instalaron data loggers en distintas altitudes 
Figura 1

LOCALIZACIÓN DEL ÁREA DE ESTUDIO
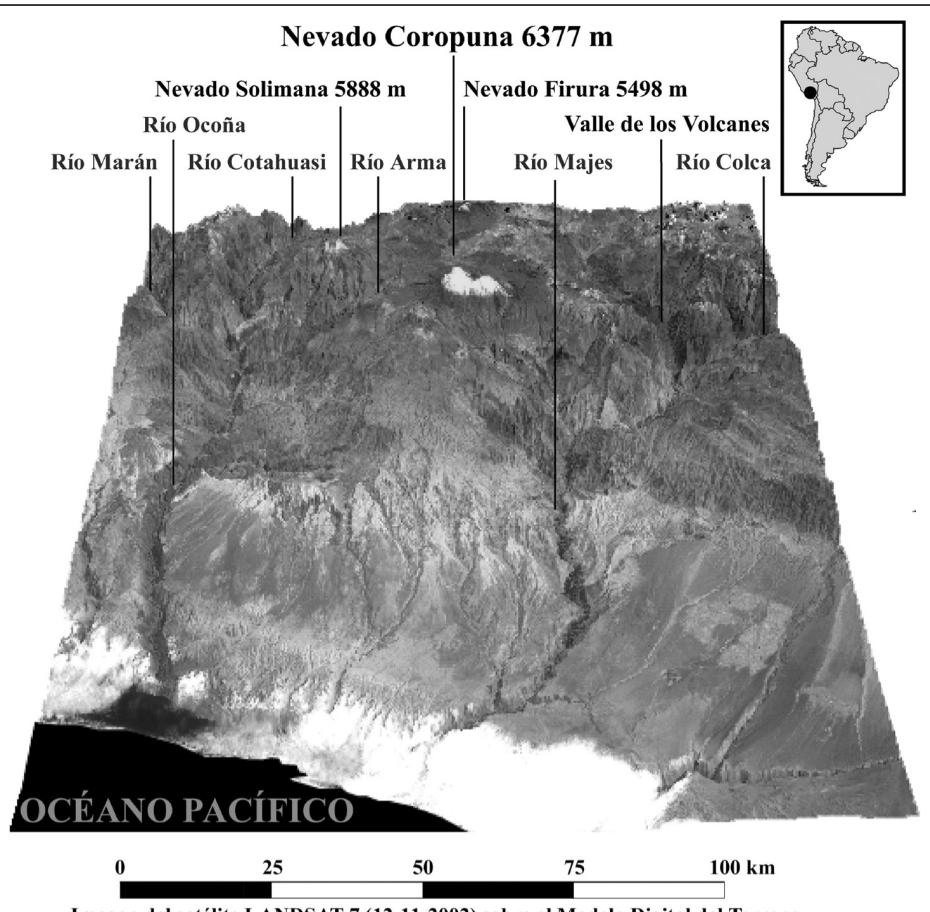

Imagen del satélite LANDSAT 7 (12-11-2002) sobre el Modelo Digital del Terreno

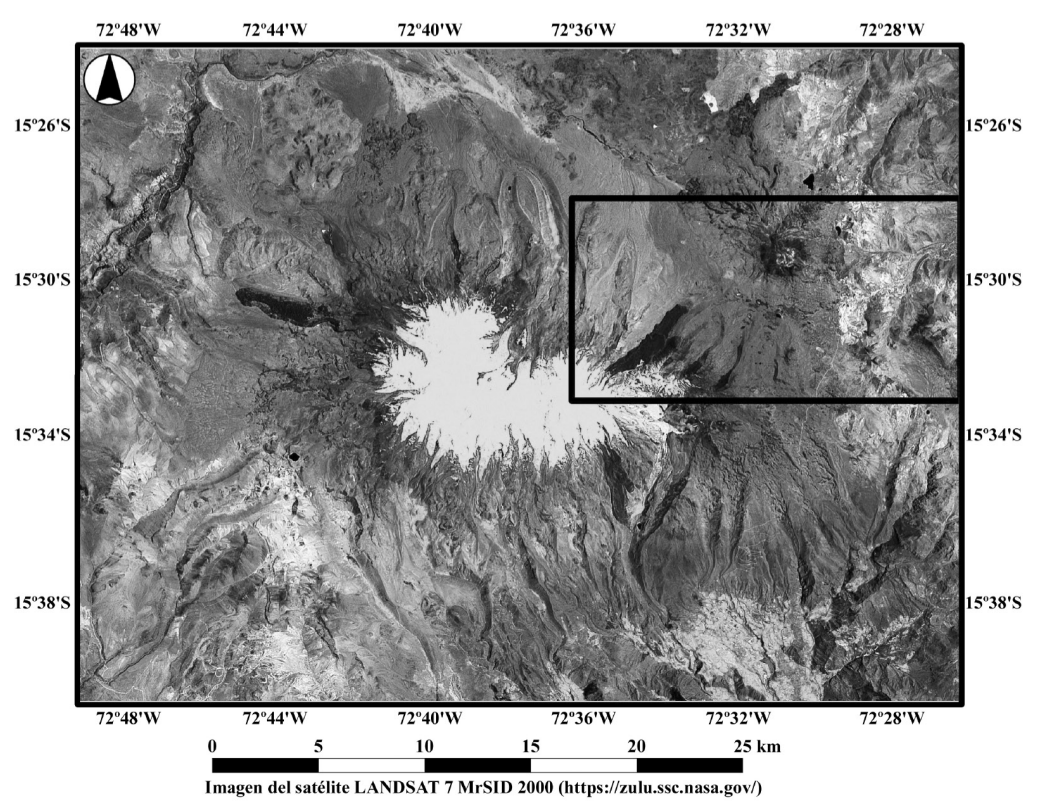

Imagen del satélite LANDSAT 7 MrSID 2000 (https://zulu.ssc.nasa.gov/) 
MAPA GEOMORFOLÓGICO

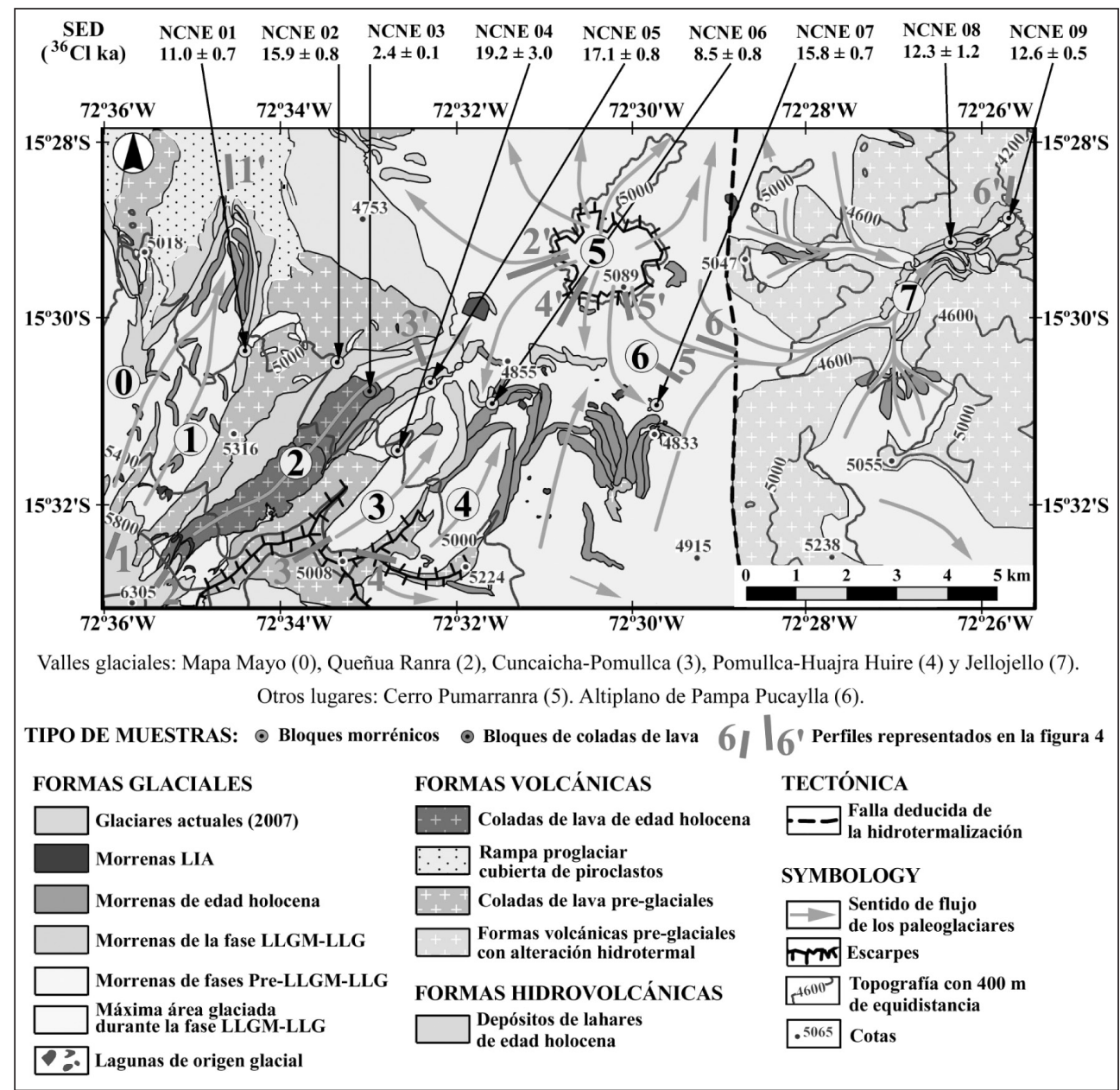

del sector NE del complejo volcánico. El análisis estadístico de varias decenas de miles de registros ha permitido elaborar modelos que localizan la isoterma anual de $0^{\circ} \mathrm{C}$ a $5350 \mathrm{msnm}$ (Úbeda, 2010). Ese dato se dedujo extrapolando el promedio anual de la temperatura del aire $\left(3,1^{\circ} \mathrm{C}\right)$ registrado a $4886 \mathrm{msnm}$, utilizando un gradiente térmico vertical basado en los registros de los data loggers y su desnivel. Además se estimó un valor de $485 \mathrm{~mm}$ para la precipitación total anual en la misma altitud, extrapolando el promedio $(364 \mathrm{~mm})$ de la serie 1965-2003 observada en la estación de Andagua (15²9’ S, 72²0’ W, 3587 m), 20 km hacia el este. Para elaborar el modelo de la precipitación se utilizó el mismo gradiente altitudinal $(0,1$ $\mathrm{mm} / \mathrm{m}$ ) usado por Klein et al 1999 en su reconstrucción regional de ELAs y paleoELAs. Su aplicación en Andagua indicó una precipitación total anual de 359 mm, con un error de sólo 
-5 mm con respecto al valor registrado en realidad. El modelo indicó un valor de $484 \mathrm{~mm}$ en la estación que se encuentra a 4886 msnm, al NE del Nevado Coropuna.

Que las ELAs y paleoELAs se incrementan de norte a sur y de este a oeste de los Andes Centrales, en el mismo sentido que la aridez del clima, es un hecho que fue señalado por Clapperton (1993) y ha confirmado más recientemente la amplia recopilación de Mark et al (2005). Debido a esa tendencia regional las ELAs y paleoELAs son bastante más altas en el Nevado Coropuna que en regiones que se encuentran al norte y al este del altiplano, como la Cordillera Blanca y las cordilleras orientales de Perú y Bolivia. En el sector SW del complejo volcánico Dornbusch (2002) estimo valores de $5558 \mathrm{~m}$ para la ELA actual y $4750 \mathrm{~m}$ para la paleoELA del LGM. En un trabajo más reciente y exhaustivo (Bromley et al., 2011a) estudiaron numerosos valles alrededor del Coropuna, sugiriendo entre $4663 \pm 232$ y $5200 \pm 68 \mathrm{~m}$ para la actualidad, $5319 \pm 139$ y $5757 \pm 52 \mathrm{~m}$ para el LLG y $4663 \pm 232$ y $5200 \pm 88 \mathrm{~m}$ para el LLGM, dependiendo de la orientación de las vertientes. En otra publicación, Ubeda (2010) ha utilizado procedimientos estadísticos para reconstruir las ELAs y paleoELAs de dos docenas de valles de los sectores NE y SE del complejo volcánico. Sus resultados indican pares de valores para ambas vertientes (NE/SE) de 5968/5862 m en 2007, 5929/5853 m en 1986, 5923/5787 m en 1955, 5886/5776 m en la LIA y 5179/4951 m durante los máximos avances del periodo LLGM-LLG.

\section{METODOLOGÍA}

La elaboración previa de una cartografía geomorfológica en un Sistema de Información Geográfica (figura 2), diferenciando unidades de origen volcánico y unidades de origen glaciar, ha proporcionado a la investigación un instrumento de análisis muy eficaz. La digitalización del mapa se apoyó en la interpretación de fotografías aéreas y ortofotos de 1955 y 1986, imágenes de los satélites LANDSAT (2000) y ASTER (2007) y seis campañas de trabajo de campo (2004-2010). Con esa herramienta y los criterios propuestos por Zreda \& Phillips (2000) se seleccionaron bloques que podía suponerse que conservaban su emplazamiento original, cuyas superficies no estaban afectadas por procesos erosivos demasiado intensos y no habían estado cubiertas por sedimentos.

Los bloques tenían un tamaño $>1$ x 1 x $1 \mathrm{~m}$ y estaban en la cresta de las morrenas o en el dorso de la colada de lava. La intensidad de la erosión se cuantificó en el campo para aplicar en el laboratorio las medidas de corrección necesarias para determinar las SED. Para extraer las muestras las superficies de las unidades geomorfológicas se golpearon con maza y cincel hasta completar $1 \mathrm{~kg}$ de roca. Posteriormente las muestras se limpiaron, molieron y tamizaron para seleccionar $200 \mathrm{~g}$ del intervalo granulométrico de las arenas $(0,85-0,15 \mathrm{~mm})$, cuyo análisis permitió determinar su composición química y la cantidad de átomos de ${ }^{36} \mathrm{Cl}$. Siguiendo los procedimientos descritos en Zreda et al. (1999) y Phillips (2003), las muestras se disolvieron en una mezcla caliente de ácidos fluorhídrico y nítrico y el cloro precipitó en forma de cloruro de plata $(\mathrm{AgCl})$. La disolución fue centrifugada y se filtró pasándola a través de una membrana de teflón de un micrón de espesor $(0,001 \mathrm{~mm})$. Las muestras resultantes se pesaron y se separó una parte que se colocó en un soporte de cobre cerrado al vacío

y se introdujo debajo de la fuente de iones de un acelerador de partículas. La espectrometría de masas permitió elaborar el recuento de los átomos de cloro. Además se determinó la presencia de elementos mayores (\%) y elementos traza (ppm). 
Las SED se calcularon mediante el tratamiento de los resultados obtenidos en el laboratorio en la aplicación online del CRONUS EARTH PROJECT (http://www.cronuscalculators. nmt.edu/cl-36/). En primer lugar se calcularon las edades de exposición utilizando los cinco modelos de escala de CRONUS (LSI, LSD, DAZ, LFT y DUN). A continuación se seleccionaron los promedios de esas cronologías y se seleccionaron como mejores estimaciones por considerarse estadísticamente más representativas. Para afrontar la discusión de los resultados y su contextualización regional se generó un cronograma paleoclimático usando los promedios cada 1 ka de la paleotemperatura de la superficie del mar en las Islas Galápagos (Lea et al., 2006) y el porcentaje (\%) de plancton de agua dulce inscrito en los sedimentos del Lago Titicaca (Fritz et al., 2007). Las bases de datos originales están disponibles en el enlace del NOAA Paleoclimatology Program (http://www.ncdc.noaa.gov/paleo/).

\section{CONTEXTO GEOMORFOLÓGICO DE LAS MUESTRAS}

Las vertientes septentrionales del Nevado Coropuna enlazan con la superficie del altiplano de Pucuncho, cuya presencia impuso durante el Pleistoceno un límite topográfico al descenso de la paleoELA. La dinámica de los paleoglaciares y la deposición de las morrenas en ese sector ha estado controlada por la subsidencia tectónica diferencial de los bloques que compartimentan el complejo volcánico (Úbeda, 2010). Por esa razón, a medida que se incrementó la depresión de la paleoELA gran parte de las masas de hielo procedentes del área de cumbres se dirigieron hacia el NW, donde la subsidencia es mayor. Ese fue el caso de los paleoglaciares que se canalizaron por los valles Mapa Mayo y Santiago (figura 2) y depositaron morrenas tan grandes que pueden reconocerse en imágenes de satélite de resolución media. Sin embargo, en la parte más oriental, las lenguas de hielo que descendieron por el interior de los valles Queñua Ranra, Cuncaicha-Pomullca y Pomullca-Huajra Huire colisionaron frontalmente en un área endorreica con los paleoglaciares que procedían del cerro Pumarranra, un edificio volcánico con forma de meseta y una altitud media de $5050 \mathrm{~m}$ que se eleva sobre el altiplano al NE del Nevado Coropuna (figura 2). La menor subsidencia de ese sector del complejo volcánico y la amplia isoaltitud de su área de cumbres, intensamente modelada por las masas de hielo, favorecieron un eficaz funcionamiento de la fábrica glaciar en el Cerro Pumarranra.

La colisión de los paleoglaciares ha quedado registrada en el amplio y complejo conjunto morrénico depositado en el altiplano. Finalmente, el descenso de la paleoELA generó un extenso campo de hielo que cubrió la mayor parte del altiplano y se desbordó por su límite NE. Durante el Holoceno reciente el edificio NE del Nevado Coropuna emitió una colada de lava de 7 km de longitud que se encauzó por el interior del valle Queñua Ranra. La fusión repentina de parte de las masas de hielo como consecuencia de la erupción generó un lahar que descendió por el valle, rompió la morrena lateral occidental del periodo LLGM-LLG y se alejó 16 km hacia el NW.

El reconocimiento del sector ha permitido delimitar numerosas formas morrénicas cuya interpretación es compleja. En la leyenda del mapa geomorfológico (figura 2) se han clasificado de la siguiente manera:

1) Las formas más recientes se han atribuido a la LIA. Aparecen inmediatamente por delante de los glaciares actuales en las cabeceras de los valles Mapa Mayo, Santiago y Que- 
ñua Ranra, en el sector NE del área de cumbres. En la parte inferior de todos los valles hay morrenas laterales de gran tamaño con un excelente grado de conservación. Su aspecto es muy parecido al de las morrenas que delimitan la máxima expansión glaciar en otras montañas de los Andes Centrales, donde se han atribuido a fases LLGM o LLG.

2) En los sectores más elevados las morrenas LLGM-LLG se apoyan en las laderas de la montaña, pero aguas abajo conforman por si solas las vertientes de los valles glaciares, que apenas han incidido en el altiplano (figura 2). La excepción es el valle Jellojello que aparece encajado en el altiplano varios cientos de metros y desemboca en el Valle de los Volcanes cerca de la localidad de Andagua. En el sector inferior de Jellojello las morrenas laterales se adosan a las vertientes y un conjunto de arcos morrénicos señalan la mínima altitud ( 4200 msnm) alcanzada por los paleoglaciares al norte del Nevado Coropuna.

3) Las unidades situadas altitudes comprendidas entre los niveles LIA y LLG-LLGM se han clasificado genéricamente como morrenas holocenas.

Para conocer la cronología de los avances de las masas de hielo se recogieron muestras (figura 3) de las superficies de bloques de las morrenas laterales de los valles Santiago (NCNE01) y Queñua Ranra (NCNE02 y NCNE05), cuyas cabeceras superan los 6000 msnm y en la actualidad están cubiertas por glaciares. También se muestrearon bloques de las morrenas de los valles Cuncaicha-Pomullca (NCNE04) y Pommullca-Huajra Huire (NCNE06), que se abren en la base septentrional de la estructura de un edificio parcialmente desmantelado que se encuentra al este de la cumbre más oriental del Nevado Coropuna, tiene altitudes más reducidas y en el presente carece de masas de hielo. Otras muestras proceden de la superficie de un bloque de un arco morrénico frontal depositado en el altiplano por la progresión de los paleoglaciares del Cerro Pumaranra (NCNE7) y de las morrenas laterales y el arco morrénico frontal más avanzado del valle Jellojello (NCNE08 y NCNE09), que fueron depositadas por las lenguas de hielo procedentes del NE del altiplano. Complementariamente se recogió una muestra en un bloque de la colada de lava holocena del valle Queñua Ranra (NCNE03).

\section{DATACIONES POR EDADES DE EXPOSICIÓN (SED)}

Con los criterios de selección indicados se recogieron las muestras cuya localización y datos de campo se resumen en la tabla 1. En la tabla 2 se indican los factores de corrección aplicados por CRONUS para corregir los efectos de la latitud y la elevación en la radiación cósmica, y conocer las tasas de producción de cosmonucleidos por reacciones de espalación, captura de muones lentos y baja energía.

En la tabla 3 se muestran las tasas de erosión, calculadas por el cociente de la erosión medida en el campo para cada muestra y la estimación de su SED preliminar y otros parámetros indicados por la aplicación online CRONUS.

La tabla 4 es un resumen de las dataciones por exposición de superficies (SED) sugeridas por CRONUS para cada modelo de escala, y el promedio de todas las estimaciones, que en este trabajo se ha seleccionado como edad más aproximada. El error proviene de la indeterminación en el recuento de átomos de ${ }^{36} \mathrm{Cl}$ cometida por el acelerador de partículas. 
Figura 3

FOTOGRAFÍAS DE LOS LUGARES DONDE SE RECOGIERON LAS MUESTRAS

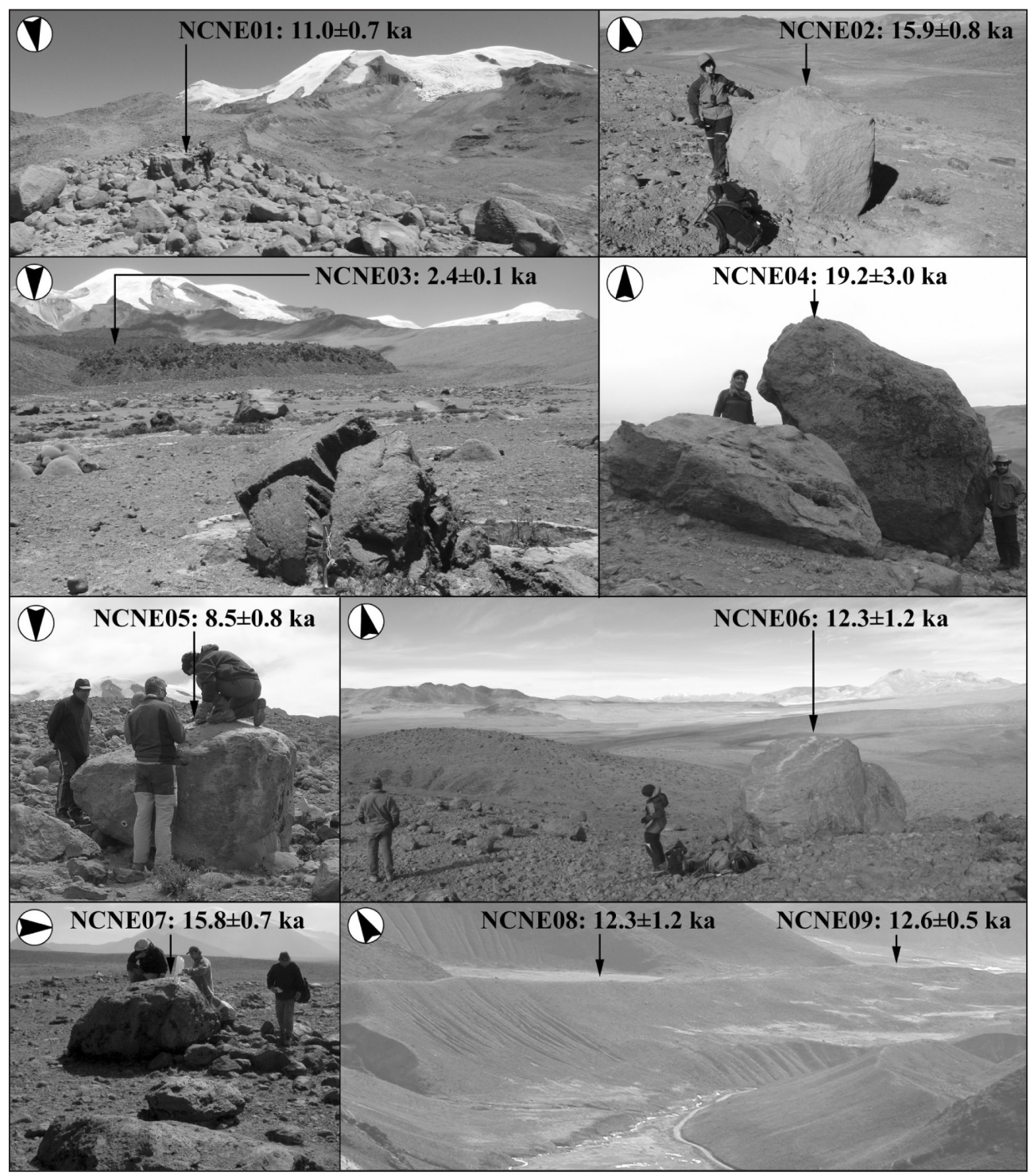


Tabla 1

DATOS DE CAMPO DE LAS MUESTRAS

\begin{tabular}{|l|c|c|c|c|}
\hline \multirow{2}{*}{ Muestra } & Latitud sur & Longitud oeste & Altitud & Presión atmosférica $^{1}$ \\
\cline { 2 - 5 } & \multicolumn{2}{|c|}{ (grados centesimales) } & $(\mathrm{msnm})$ & $(\mathrm{mb})$ \\
\hline NCNE01 & $-15,50$ & $-72,58$ & 5060 & 553 \\
\hline NCNE02 & $-15,51$ & $-72,56$ & 5013 & 556 \\
\hline NCNE03 & $-15,51$ & $-72,55$ & 4901 & 564 \\
\hline NCNE04 & $-15,51$ & $-72,54$ & 4915 & 563 \\
\hline NCNE05 & $-15,52$ & $-72,55$ & 5052 & 554 \\
\hline NCNE06 & $-15,51$ & $-72,53$ & 4929 & 562 \\
\hline NCNE07 & $-15,54$ & $-72,49$ & 4914 & 563 \\
\hline NCNE08 & $-15,48$ & $-72,43$ & 4080 & 622 \\
\hline NCNE09 & $-15,48$ & $-72,45$ & 4384 & 601 \\
\hline \multirow{3}{*}{ Muestra } & Erosión de la & Espesor de la & Densidad de la & Efecto del relieve \\
\cline { 2 - 5 } & superficie & muestra & muestra & sobre la radiación \\
\hline NCNE01 & $(\mathrm{cm})$ & $(\mathrm{cm})$ & 2,5 & factor adimensional) $\left.^{3}\right)$ \\
\hline NCNE02 & 2,0 & 1,5 & 2,3 & 0,9937 \\
\hline NCNE03 & 2,5 & 2,0 & 2,2 & 0,9957 \\
\hline NCNE04 & 0,0 & 3,0 & 2,5 & 0,9941 \\
\hline NCNE05 & 0,0 & 2,5 & 2,6 & 0,9886 \\
\hline NCNE06 & 2,5 & 2,5 & 2,8 & 0,9934 \\
\hline NCNE07 & 2,5 & 2,5 & 2,5 & 0,9977 \\
\hline NCNE08 & 2,0 & 2,5 & 2,5 & 0,9977 \\
\hline NCNE09 & 4,2 & 4,0 & 2,3 & 0,9947 \\
\hline
\end{tabular}

${ }^{1}$ Los datos de presión atmosférica se han calculado utilizando un gradiente altitudinal deducido de los calculados por Bromley et al (2009).

Tabla 2

FACTORES DE CORRECCIÓN DE LOS EFECTOS DE LAALTITUD Y LA ELEVACIÓN Y TASA DE PRODUCCIÓN DE COSMONUCLEIDOS (ESTIMADOS EN CRONUS)

\begin{tabular}{|c|c|c|c|c|c|c|c|c|c|}
\hline \multirow{2}{*}{$\begin{array}{c}\text { Código } \\
\text { Muestra }\end{array}$} & $\begin{array}{c}\text { Factores de } \\
\text { corrección de } \\
\text { altitud y elevación }\end{array}$ & \multicolumn{5}{|c|}{ Tasa de producción de cosmonucleidos (átomos/gramo/año) } \\
\cline { 2 - 11 } & $\begin{array}{c}\text { Para } \\
\text { neutrones }\end{array}$ & $\begin{array}{c}\text { Para } \\
\text { muones }\end{array}$ & $C a$ & $K$ & $F e$ & $T i$ & $C a$ & $K$ & $C l$ \\
\hline NCNE01 & 12,61 & 4,32 & 25,11 & 48,39 & 0,76 & 0,30 & 0,73 & 1,07 & 12,33 \\
\hline NCNE02 & 12,37 & 4,26 & 23,74 & 46,93 & 0,79 & 0,29 & 0,70 & 1,05 & 11,06 \\
\hline NCNE03 & 11,80 & 4,12 & 22,39 & 45,85 & 0,81 & 0,32 & 0,67 & 1,05 & 87,79 \\
\hline NCNE04 & 12,57 & 4,31 & 28,48 & 44,21 & 0,98 & 0,36 & 0,84 & 0,99 & 35,17 \\
\hline NCNE05 & 11,87 & 4,14 & 27,52 & 42,14 & 0,94 & 0,35 & 0,82 & 0,96 & 37,28 \\
\hline NCNE06 & 11,94 & 4,16 & 25,41 & 43,84 & 0,90 & 0,35 & 0,76 & 1,00 & 13,63 \\
\hline NCNE07 & 11,54 & 4,06 & 28,08 & 37,05 & 1,05 & 0,41 & 0,84 & 0,85 & 90,89 \\
\hline NCNE08 & 8,16 & 3,19 & 9,63 & 34,35 & 0,40 & 0,10 & 0,33 & 0,89 & 16,53 \\
\hline NCNE09 & 9,39 & 3,52 & 8,36 & 44,00 & 0,40 & 0,11 & 0,27 & 1,07 & 35,37 \\
\hline
\end{tabular}


Tabla 3

TASA DE EROSIÓN Y OTROS PARÁMETROS INDICADOS POR CRONUS

\begin{tabular}{|c|c|c|c|c|c|c|c|c|}
\hline \multirow{2}{*}{$\begin{array}{c}\text { Código } \\
\text { Muestra }\end{array}$} & \multirow{2}{*}{$\begin{array}{c}\text { Tasa de } \\
\text { erosión }\end{array}$} & \multicolumn{7}{|c|}{ Otros parámetros indicados por CRONUS } \\
\cline { 2 - 9 } & $(\mathrm{mm} / \mathrm{ka})$ & $\boldsymbol{\Sigma}_{\text {th.ss }}$ & $\boldsymbol{\Sigma}_{\text {eth.s }}$ & $\boldsymbol{\Sigma}_{\text {sc.ss }}$ & $\mathbf{Q}_{\mathrm{s}}$ & $\mathbf{Q}_{\text {th }}$ & $\mathbf{Q}_{\text {eth }}$ & $\mathbf{Q}_{\text {eth }}$ \\
\hline NCNE01 & 0,17 & 0,006 & 0,013 & 0,094 & 0,995 & 1,076 & 1,070 & 0,999 \\
\hline NCNE02 & 0,14 & 0,006 & 0,013 & 0,094 & 0,992 & 1,096 & 1,082 & 0,999 \\
\hline NCNE03 & 0,00 & 0,006 & 0,013 & 0,095 & 0,986 & 1,127 & 1,113 & 0,998 \\
\hline NCNE04 & 0,00 & 0,006 & 0,013 & 0,095 & 0,987 & 1,122 & 1,108 & 0,998 \\
\hline NCNE05 & 0,11 & 0,006 & 0,013 & 0,094 & 0,987 & 1,122 & 1,112 & 0,998 \\
\hline NCNE06 & 0,26 & 0,007 & 0,013 & 0,095 & 0,986 & 1,128 & 1,121 & 0,998 \\
\hline NCNE07 & 0,00 & 0,007 & 0,013 & 0,095 & 0,988 & 1,123 & 1,111 & 0,998 \\
\hline NCNE08 & 0,15 & 0,006 & 0,013 & 0,093 & 0,976 & 1,201 & 1,162 & 0,997 \\
\hline NCNE09 & 0,30 & 0,005 & 0,013 & 0,094 & 0,989 & 1,102 & 1,082 & 0,999 \\
\hline
\end{tabular}

Tabla 4

DATACIONES POR EXPOSICIÓN DE SUPERFICIES (SED)

\begin{tabular}{|c|l|l|c|c|c|c|c|c|c|c|c|c|c|c|}
\hline \multicolumn{3}{|c|}{ MUESTRAS } & \multicolumn{2}{c|}{ MEDIA } & \multicolumn{2}{|c|}{ LSI } & \multicolumn{2}{|c|}{ LSD } & \multicolumn{2}{|c|}{ DAZ } & \multicolumn{2}{|c|}{ LFT } & \multicolumn{2}{|c|}{ DUN } \\
\hline Muestra & \multicolumn{1}{|c|}{ Sitio } & Contexto & $\mathbf{k a}$ & $\mathbf{\pm}$ & $\mathrm{ka}$ & \pm & $\mathrm{ka}$ & \pm & $\mathrm{ka}$ & \pm & $\mathrm{ka}$ & \pm & $\mathrm{ka}$ & \pm \\
\hline NCNE01 & $\begin{array}{l}\text { Valle } \\
\text { Santiago }\end{array}$ & $\begin{array}{l}\text { Bloque } \\
\text { morrénico }\end{array}$ & $\mathbf{1 1 , 0}$ & $\mathbf{0 , 7}$ & 12,1 & 0,7 & 12,0 & 0,7 & 10,1 & 0,5 & 10,5 & 0,7 & 10,3 & 0,6 \\
\hline NCNE02 & $\begin{array}{l}\text { Queñua } \\
\text { Ranra valley }\end{array}$ & $\begin{array}{l}\text { Bloque } \\
\text { morrénico }\end{array}$ & $\mathbf{1 5 , 9}$ & $\mathbf{0 , 8}$ & 17,4 & 1,0 & 16,8 & 0,8 & 14,8 & 0,8 & 15,4 & 0,8 & 15,0 & 0,8 \\
\hline NCNE03 & $\begin{array}{l}\text { Quenua } \\
\text { Ranra valley }\end{array}$ & $\begin{array}{l}\text { Colada de } \\
\text { lava }\end{array}$ & $\mathbf{2 , 4}$ & $\mathbf{0 , 1}$ & 2,5 & 0,2 & 2,7 & 0,2 & 2,3 & 0,0 & 2,5 & 0,2 & 2,1 & 0,0 \\
\hline NCNE04 & $\begin{array}{l}\text { C. Pomullca } \\
\text { valley }\end{array}$ & $\begin{array}{l}\text { Bloque } \\
\text { morrénico }\end{array}$ & $\mathbf{1 9 , 2}$ & $\mathbf{3 , 0}$ & 22,0 & 3,8 & 20,5 & 3,3 & 17,7 & 2,6 & 18,1 & 2,6 & 18,0 & 2,8 \\
\hline NCNE05 & $\begin{array}{l}\text { Queñua } \\
\text { Ranra valley }\end{array}$ & $\begin{array}{l}\text { Bloque } \\
\text { morrénico }\end{array}$ & $\mathbf{1 7 , 1}$ & $\mathbf{0 , 8}$ & 19,3 & 0,7 & 18,2 & 0,7 & 15,7 & 0,4 & 16,1 & 0,4 & 16,1 & 1,8 \\
\hline NCNE06 & $\begin{array}{l}\text { P. Huajra } \\
\text { Huire valley }\end{array}$ & $\begin{array}{l}\text { Bloque } \\
\text { morrénico }\end{array}$ & $\mathbf{8 , 5}$ & $\mathbf{0 , 8}$ & 9,5 & 0,8 & 9,4 & 0,8 & 7,8 & 0,7 & 8,2 & 0,8 & 7,9 & 0,7 \\
\hline NCNE07 & $\begin{array}{l}\text { Pampa } \\
\text { Pucaylla }\end{array}$ & $\begin{array}{l}\text { Bloque } \\
\text { morrénico }\end{array}$ & $\mathbf{1 5 , 8}$ & $\mathbf{0 , 7}$ & 18,0 & 0,9 & 17,0 & 0,7 & 14,5 & 0,6 & 14,7 & 0,6 & 14,7 & 0,7 \\
\hline NCNE08 & Jellojello 1 & $\begin{array}{l}\text { Bloque } \\
\text { morrénico }\end{array}$ & $\mathbf{1 2 , 3}$ & $\mathbf{1 , 2}$ & 12,9 & 1,3 & 12,7 & 1,2 & 11,7 & 1,1 & 12,2 & 1,1 & 11,8 & 1,1 \\
\hline NCNE09 & Jellojello 2 & $\begin{array}{l}\text { Bloque } \\
\text { morrénico }\end{array}$ & $\mathbf{1 2 , 6}$ & $\mathbf{0 , 5}$ & 13,6 & 0,6 & 13,3 & 0,5 & 11,9 & 0,6 & 12,2 & 0,5 & 12,0 & 0,5 \\
\hline
\end{tabular}


Las SED calculadas por CRONUS variaron entre 0,6 y $4,3{ }^{36} \mathrm{Cl} \mathrm{ka}$, con una desviación media de 2,4 ${ }^{36} \mathrm{Cl} \mathrm{ka}$. Atendiendo a la interpretación de su significado geomorfológico, las cronologías pueden agruparse en cinco conjuntos:

1) Las muestras NCNE $04\left(19.2 \pm 3.0{ }^{36} \mathrm{Cl} \mathrm{ka}\right)$; NCNE $05\left(17.1 \pm 0.8{ }^{36} \mathrm{Cl} \mathrm{ka}\right)$; NCNE 02 $\left(15.9 \pm 0.8{ }^{36} \mathrm{Cl} \mathrm{ka}\right)$ y NCNE $07\left(15.8 \pm 0.7{ }^{36} \mathrm{Cl} \mathrm{ka}\right)$ indican la sucesión de una o varias fases de máxima expansión glaciar inicial entre 22 y $16 \mathrm{ka}$ antes del presente. Durante ese periodo las masas de hielo ocuparon los valles que se abren en las vertientes de los edificios del Nevado Coropuna y el altiplano, y probablemente también alcanzaron el fondo del valle Jellojello, donde las formas morrénicas muestreadas se superponen a otras anteriores.

2) Otro conjunto de SED reflejan una fase de máxima expansión final entre 13 y 11 ka, durante la que los paleoglaciares procedentes del NE del altiplano generaron los arcos morrénicos del valle Jellojello, de los que proceden las muestras NCNE $08\left(12.3 \pm 1.2{ }^{36} \mathrm{Cl} \mathrm{ka}\right)$ y NCNE $09\left(12.6 \pm 0.5{ }^{36} \mathrm{Cl} \mathrm{ka}\right)$. Debido a que representan la menor altitud alcanzada por los flujos en el sector NE del Nevado Coropuna, de su presencia se deduce que en esa época las masas de hielo también debían ocupar el altiplano y los valles que se abren en las vertientes de los edificios volcánicos.

3) En el valle Santiago sucedió un último máximo avance en $11.0 \pm 0.7{ }^{36} \mathrm{Cl}$ ka (muestra NCNE 01), que depositó morrenas de gran tamaño que se superponen a otras más viejas.

4) Las evidencias geomorfológicas demuestran que con posterioridad al periodo LLGMLLG debió desencadenarse una fase de rápida deglaciación hasta el nivel del altiplano. La presencia de numerosas morrenas entre las altitudes de las fases LLGM-LLG y LIA demuestra que durante el Holoceno sucedieron reavances de menor entidad, probablemente vinculados con oscilaciones de la precipitación. Una parte de esas formas se apoyan unas sobre otras, sugiriendo que fueron depositados por una secuencia rápida de pulsos positivos y negativos. La muestra NCNE 05 se recogió en una morrena lateral que inicialmente se consideró que podría atribuirse a la fase LLGM-LLG, pero su SED $\left(8.5 \pm 0.8{ }^{36} \mathrm{Cl} \mathrm{ka}\right)$ indica que debe incluirse en el grupo de morrenas holocenas. Fue depositada por una pulsación que desplazó parte de las morrenas preexistentes, sin alcanzar la máxima extensión anterior, representada por un arco morrénico frontal depositado por la colisión de paleoglaciares durante el LLGM-LLG.

5) Finalmente, la SED de la colada de lava del valle Queñua Ranra (muestra NCNE 03, $2.4 \pm 0.1{ }^{36} \mathrm{Cl} \mathrm{ka}$ ) confirma un hecho que previamente habían sugerido su estado de conservación y la interpretación de su contexto geomorfológico: la reactivación de la actividad volcánica en el Holoceno reciente, cuando los frentes de las lenguas de hielo alcanzaron posiciones próximas a las actuales.

Como complemento a la explicación del contexto de las muestras se han dibujado los perfiles topográficos que se muestran en la figura 4, correspondientes a los que se han señalado previamente en el mapa geomorfológico (figura 2). Sobre las vertientes de los edificios volcánicos se han representando las masas de hielo actuales, las formas morrénicas generadas por la progresión de los paleoglaciares desde el LLGM-LLG y la colada de lava que durante el Holoceno reciente se canalizó por el valle Queñua Ranra, con indicación de las SED correspondientes a cada muestra. 


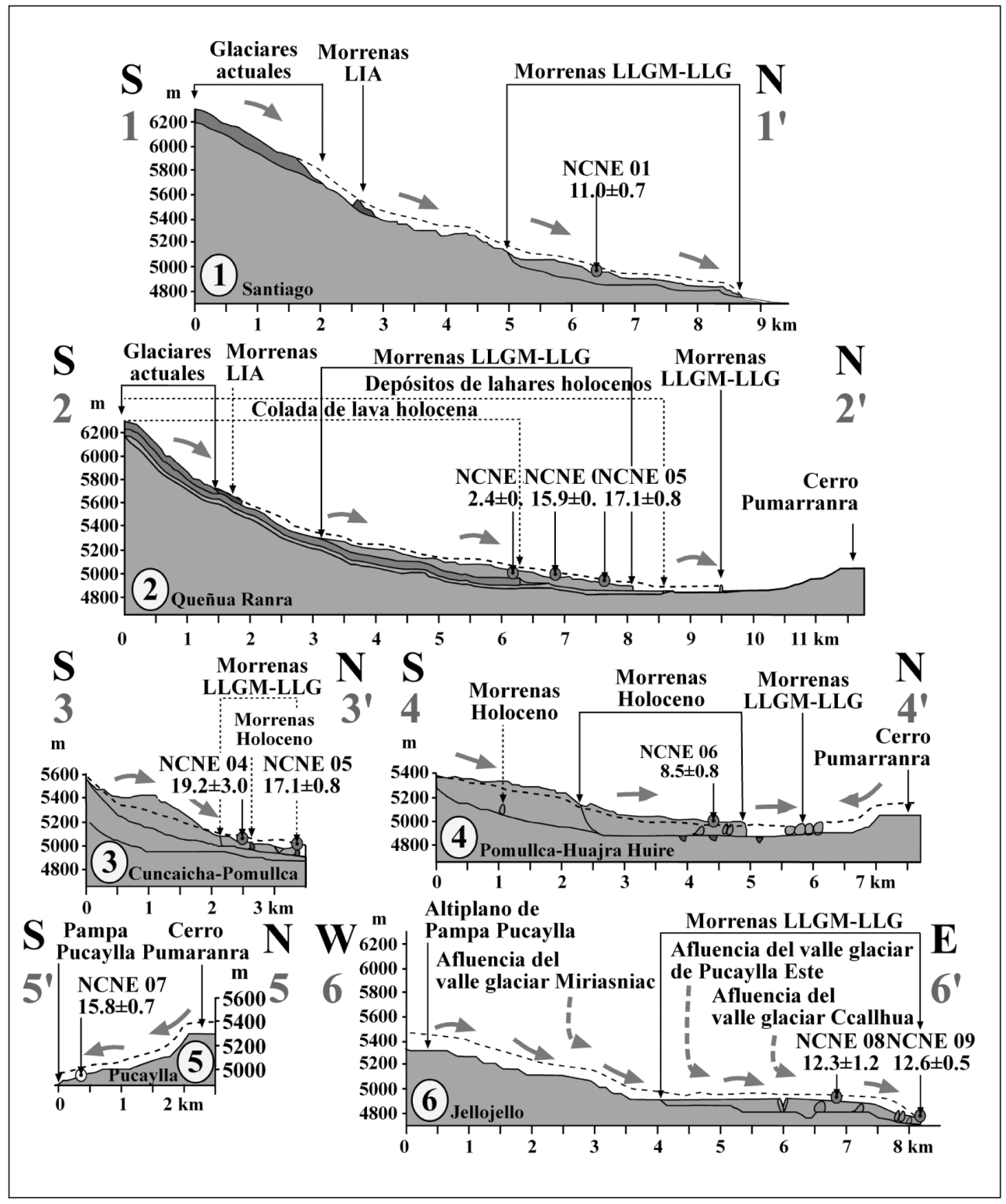




\section{DISCUSIÓN}

Las SED que se han presentado en este trabajo, deducidas de la acumulación de ${ }^{36} \mathrm{Cl}$, y parte de las que establecieron Bromley et al (2009) midiendo la presencia de ${ }^{3} \mathrm{He}$ en la superficie de las rocas, proporcionan un cronograma de la máxima expansión del sistema glaciar del Nevado Coropuna durante el LLGM-LLG. Para contrastar las SED de las morrenas de los valles del sector NE del complejo volcánico con las cronologías glaciales de los Andes Centrales es necesario tener en cuenta que las dataciones cosmogénicas de la cordillera presentan una notable dispersión, a causa de tres factores: 1) El procedimiento aplicado para calcular las edades de exposición; 2) La evolución paleoclimática, que controla la elevación y depresión de la ELA. 3) La dinámica experimentada localmente por los paleoglaciares en función de su contexto geomorfológico.

Con respecto al procedimiento empleado para estimar las SED, conviene señalar que debido a su carácter reciente los métodos de aplicación de las técnicas cosmogénicas no están todavía suficientemente estandarizados. El CRONUS EARTH PROJECT (www.physics.purdue.edu/www.cronus-eu.net) representa una oportunidad para homogeneizar los procedimientos. Permite aplicar los factores de corrección específicos de cada muestra (espesor y geometría de la superficie y sombra topográfica) y los que se deducen de la dependencia de la tasa de producción local de la latitud y la altitud, a causa de la desviación geomagnética y la atenuación de la radiación cósmica cuando pasa a través de la atmósfera.

Zech et al (2008) utilizaron la herramienta online CRONUS para ${ }^{10} \mathrm{Be}$ (Balco et al 2008) para recalcular SED publicadas en trabajos anteriores: 1) Farber et al (2005) en cuatro valles de la Cordillera Blanca $\left.\left(9^{\circ} \mathrm{S}, 77^{\circ} \mathrm{W}\right) ; 2\right)$ Smith et al $(2005 \mathrm{~b}, \mathrm{c})$ en la región de Junín $\left(11^{\circ} \mathrm{S}\right.$, $\left.76^{\circ} \mathrm{W}\right)$ y los valles Milluni y Zongo, de la Cordillera Real $\left(16^{\circ} \mathrm{S}, 68^{\circ} \mathrm{W}\right)$; y 3$)$ Zech et al (2007) en el Valle San Francisco $\left(16^{\circ} \mathrm{S}, 68^{\circ} \mathrm{W}\right)$, también en la Cordillera Real, y los valles del río Suturi y Huara Loma $\left(17^{\circ} \mathrm{S}, 66^{\circ} \mathrm{W}\right)$, en la región de Cochabamba.

Zech et al (2008) correlacionaron las morrenas atribuidas al LLGM en la Cordillera Blanca (grupo Rurec), la región del Lago Junín y el valle Milluni (grupos C), y los valles San Francisco, río Suturi y Huara Loma. En su discusión consideran que las SED de los Andes tropicales áridos, donde el promedio de la precipitación total anual es $<700 \mathrm{~mm}$, sugieren un patrón global y coherente para las fases LLGM (entre $30 / 27$ y $23 / 20^{10} \mathrm{Be} k a$ ) y LLG (entre $17 / 15$ y $\left.12 / 10^{10} \mathrm{Be} \mathrm{ka}\right)$. Zech et al. (2008) estimaron cada par de dataciones empleando para la primera el modelo de Lal (1991) y Stone (2000), y para la segunda el modelo de Lifton (2005), que produce cronologías más recientes. Los intervalos propuestos por Zech et al (2008) tienen una notable amplitud cronológica (10 ka para las fases LLGM y 7 ka para los episodios LLG), que refleja la dispersión de las SED de diferentes lugares de los Andes Centrales.

Bromley et al (2009) intentaron minimizar las diferencias de los resultados que obtuvieron en el Nevado Coropuna con respecto a las SED de regiones más húmedas de la cordillera. Con ese objetivo aplicaron dos modelos de producción de cosmonucleidos, Lm (Lal, 1991; Nishiizumi et al., 1989; Stone, 2000) y Li (Lifton et al., 2005), para recalcular las dataciones de otras montañas, argumentando que la tasa de producción todavía no ha sido determinada con exactitud en los Andes Centrales. De ese modo aproximaron sus SED a las que habían deducido los demás investigadores, pero supeditaron la objetividad del método a los resultados que esperaban obtener. 
En trabajos más recientes (Úbeda, 2010) se ha sugerido que las asincronías glaciales podían deberse al gradiente de aridez del clima regional, hacia el sur y el oeste de los Andes Centrales. Su enfoque se ha basado en considerar que para que la expansión glaciar inicial comenzase en las regiones más áridas fue necesario que previamente la precipitación se incrementase en el mismo sentido. Es una interpretación paleoclimática coherente con las ideas de Clapperton (2000), que analizó una larga lista de cronologías glaciales de ambos hemisferios de la cordillera panamericana y concluyó que las diferencias regionales probablemente reflejan cambios pasados en la distribución de la humedad.

La hipótesis paleoclimática también es compatible con un principio señalado por Zech et al (2008): los estudios del balance de masa y las observaciones de la altitud de la línea de equilibrio (ELA) indican que cuando el promedio de la precipitación total anual es $>700$ mm los glaciares tienden a ser más sensibles a la precipitación que a la temperatura (Seltzer, 1990; Kull \& Grosjean, 2000; Kaser \& Osmaston, 2002).

La reconstrucción del modelo del balance de masa de los paleoglaciares del Nevado Coropuna durante el LLGM-LLG (Úbeda 2010), modificando el método de Klein et al (1999), ha sugerido que un incremento de un 6,4\% de la precipitación actual (494 mm/a a $4886 \mathrm{msnm}$ ), combinado con una depresión de la paleotemperatura del aire de $-6,6^{\circ} \mathrm{C}$, pudo ser suficiente para aumentar un $49 \%$ la humedad del clima, explicando la expansión glaciar que refleja el mapa geomorfológico (figura 2). El modelo anula el balance de masa a 5179 $\mathrm{m}$ de altitud, donde la ELA climática y la ELA geomorfológica se nivelan, como debió suceder cuando las masas de hielo alcanzaron su máxima expansión y se equilibraron con el clima.

Las SED de Farber et al (2005), Smith et al (2005b,c), Zech et al (2007) y Bromley et al (2009), junto con las que se han presentado en este trabajo, soportan aceptablemente la hipó-

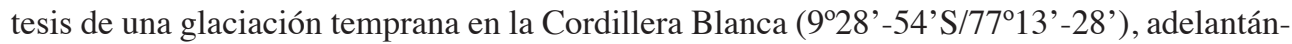
dose al LGM global y expandiéndose progresivamente hacia el sur y el oeste de los Andes Centrales. Las SED publicadas por Farber et al (2005) para el grupo Rurec, en la Cordillera Blanca $\left(\sim 2{ }^{10} \mathrm{Be} \mathrm{ka}\right)$, son contemporáneas o inmediatamente posteriores a la culminación de la progresión de los inlandsis del NE de Norteamérica y el oeste de la Antártida, y algunos glaciares de montaña del Hemisferio Norte, en $33-29{ }^{14} \mathrm{C}$ cal ka (Clark et al, 2009).

La interpretación paleoclimática también es coherente con los resultados obtenidos por Hall et al (2009) en la Cordillera Huayhuash (1005'-25'S $\left./ 76^{\circ} 45^{\prime}-55^{\prime} \mathrm{W}\right)$, donde SED de umbrales rocosos con pulimento glaciar permiten atribuir una cronología $>26^{10} \mathrm{Be}$ ka a la última máxima expansión de las masas de hielo. En aparente sincronía con el LGM global, esa fecha coincide con el mínimo nivel medio de los océanos de los últimos 50 ka, identificado en $\sim 26$ ka por tres modelos independientes (Clark et al., 2009; Lambeck \& Chappell, 2001; Yokoyama et al., 2000). El descenso del nivel del mar en $\sim 130 \mathrm{~m}$ se ha relacionado con que en ese momento los inlandsis regionales debían encontrarse en la plenitud de su crecimiento (Clark et al., 2009). El retraso con respecto a las anteriores cronologías de las SED $\left(24^{10} \mathrm{Be}\right.$ ka) que Zech et al (2007) dedujeron para la máxima expansión inicial en el valle San

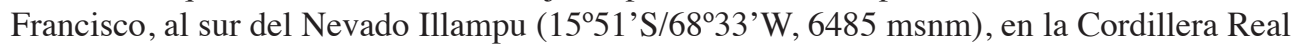
de Bolivia, podría reflejar el paulatino avance hacia el sur de condiciones notablemente más húmedas que las actuales. Posteriormente la humedad y la glaciación se habrían extendido todavía más hacia el sur, propiciando la aparición de grandes lagos en las cuencas del alti- 
plano boliviano $\left(18-20{ }^{\circ} \mathrm{S}\right)$ en $18-14{ }^{14} \mathrm{C}$ cal ka, durante el Ciclo Tauca definido por Plazcek (2005) y Plazcek et al (2006).

Las SED deducidas por Bromley et al (2009) de las muestras NC9 y NC10 del Nevado Coropuna (con un promedio de $20.9 \pm 0.7^{3} \mathrm{He} \mathrm{ka),} \mathrm{presentan} \mathrm{una} \mathrm{demora} \mathrm{con} \mathrm{respecto} \mathrm{a} \mathrm{las}$ dataciones realizadas por Zech et al (2007) en el Valle San Francisco ( $24^{10} \mathrm{Be}$ ka), sugierendo que la humedad también se incrementó progresivamente de este a oeste del altiplano. Además de retrasar el comienzo de la glaciación, la aridez provocó otras diferencias entre las regiones más húmedas y más áridas de los Andes Centrales.

En la Cordillera Blanca y la Cordillera Huayhuash la evolución de los paleoglaciares después del máximo es coherente con la tendencia global al calentamiento que refleja la SST de las Islas Galápagos (figura 5; Lea et al. 2005). En esas regiones más húmedas después de la máxima expansión inicial ocurrieron reavances de menor entidad que depositaron morrenas en altitudes cada vez más elevadas. Sin embargo en las regiones más áridas las masas de hielo experimentaron una expansión inicial seguida de varios reavances que volvieron a alcanzar una magnitud similar.

La evolución del plancton de agua dulce en el Lago Titicaca (figura 5; Fritz et al. 2007), que indica que a pesar de la tendencia al calentamiento global, el clima de los Andes Centrales debió continuar favoreciendo al avance de los paleoglaciares hasta $9{ }^{14} \mathrm{C}$ cal ka. Las edades de exposición NCNE $01\left(11.0^{36} \mathrm{Cl} \mathrm{ka}\right)$, NCNE $08\left(12.3^{36} \mathrm{Cl} \mathrm{ka}\right)$ y NCNE $09\left(12.6^{36} \mathrm{Cl} \mathrm{ka}\right)$ del Nevado Coropuna (figura 2, tabla 4) sugieren la misma posibilidad. La hipótesis es coherente con las SED de la Cordillera Real de Bolivia publicadas por Zech et al (2007) para los valles San Francisco $\left(12,9{ }^{10} \mathrm{Be}\right.$ ka), Río Suturi $\left(13,0{ }^{10} \mathrm{Be}\right.$ ka) y Huama Loma $\left(11,8^{10} \mathrm{Be} \mathrm{ka}\right)$, y Smith et al $(2011)$ para el valle Pasto Grande $\left(12,9^{10} \mathrm{Be}\right)$, en el Nevado Illimani $\left(17^{\circ} \mathrm{S}, 68^{\circ} \mathrm{W}\right)$.

La interpretación que se propone también es compatible con las SED estimadas por Bromley et al (2011b) en el Nevado Coropuna. En ese trabajo se han deducido las edades de ${ }^{3} \mathrm{He}$ de 12 muestras de las morrenas del valle Santiago, aguas arriba de los depósitos del máximo avance de las masas de hielo. Utilizando el modelo de escala de Lifton et al (2005) obtuvieron un rango de resultados entre 11.5 y $9.5{ }^{3} \mathrm{He} \mathrm{ka}$, con un promedio de $10.2{ }^{3} \mathrm{He} \mathrm{ka}$. Esas cronologías serían factibles en el escenario de una máxima expansión final seguida por un rápido proceso de deglaciación, interrumpido por pulsaciones positivas más esporádicas. Las evidencias geomorfológicas cartografiadas por Úbeda (2010) para el sector SE del Nevado Coropuna son coherentes con ese escenario. Sin embargo, Bromley et al (2011b) prefirieron reducir la tasa de producción de cosmonucleidos aplicando otros modelos de escala (Lal, 1991; Stone, 2000 y Nishiizumi et al, 1989). De ese modo incrementaron el rango de edades hasta $11.9-13.9{ }^{3} \mathrm{He} \mathrm{ka}$, con un promedio de $12.8{ }^{3} \mathrm{He} \mathrm{ka}$, y aproximaron sus resultados al LG global.

La hipótesis paleoclimática permite explicar la dispersión de parte de las SED glaciales de los Andes Centrales, pero también se han publicado otras cuya interpretación requiere incorporar en el análisis un criterio geomorfológico: si están suficientemente abastecidos por el área fuente, los reavances glaciares pueden borrar las evidencias de anteriores pulsaciones y distorsionar la interpretación del conjunto. La interpretación de algunos de los resultados presentados en este trabajo requiere la aplicación de ese enfoque geomorfológico. 
La muestra NCNE $01\left(11.0^{36} \mathrm{Cl} \mathrm{ka}\right)$ está datando un reavance en el valle Santiago que borró las evidencias anteriores, y la muestra NCNE $06\left(8.5^{36} \mathrm{Cl} \mathrm{ka}\right)$ otra fase de progresión en el valle Cuncaicha-Pomullca, que erosionó las morrenas laterales pre-existentes sin llegar a afectar a un arco morrénico frontal que se encuentra en el sector terminal del valle. El reavance que data la muestra NCNE 06 es contemporáneo a otro que Licciardi et al (2009) han descrito en la Cordillera Vilcabamba $\left(13^{\circ} \mathrm{S}, 73^{\circ} \mathrm{W}\right)$, considerándolo coherente con pulsaciones de edades semejantes en Noruega (Nesje et al, 2000) y los Alpes Austriacos (Kerschner \& Ivy-Ochs, 2008).

Lo que sucede en los valles Santiago y Cuncaicha-Pomullca, al NE del Nevado Coropuna, es habitual en muchas regiones de los Andes Centrales. En algunas montañas las morrenas depositadas por diferentes pulsaciones finipleistocenas están muy cerca unas a otras. También son frecuentes los casos en los que paleoglaciares más recientes sobrepasaron la posición alcanzada por avances anteriores, desmantelando parcial o completamente sus depósitos.

Las publicaciones de SED glaciales de los Andes Centrales describen casos de estudio inscritos en contextos geomorfológicos que sugieren la repetición de los mismos procesos. En la Cordillera Blanca, la cordillera Huayhuash y la Cordillera Real de Bolivia se han encontrado evidencias en ese sentido:

1) Las morrenas de los grupos Rurec y Laguna Baja (Cordillera Blanca), datadas por Farber et al. 2005 en 29 y $19^{10} \mathrm{Be}$ ka, están separadas sólo por unas decenas de metros.

2) En el valle de Carhuacocha (Cordillera Huayhuash), Hall et al. (2009) encontraron morrenas de $14^{10} \mathrm{Be}$ ka que casi cabalgaban a otras de $19^{10} \mathrm{Be}$ ka.

3) Zech et al. (2007) describen un fenómeno parecido en el valle de San Francisco, en la Cordillera Real, donde la morrena lateral depositada por un máximo avance glaciar tiene una SED de $19^{10} \mathrm{Be}$ ka. Valle abajo la unidad se separa en dos morrenas, con cronologías de $24{ }^{10} \mathrm{Be}$ ka la más externa y $17^{10} \mathrm{Be}$ ka la más interna. En el mismo trabajo se describe un contexto geomorfológico similar en el valle del río Suturi, en la región de Cochabamba, donde hay una morrena lateral con una edad de $13^{10} \mathrm{Be}$ ka que vertiente abajo se separa en dos unidades, con edades de $15-17^{10} \mathrm{Be}$ ka para la morrena exterior y $11-12^{10} \mathrm{Be}$ ka para la morrena interior.

4) En el valle de Wara Wara, también en la región de Cochabamba, Zech et al. (2010) encontraron una morrena lateral que ocasionalmente se divide en varias unidades que nunca llegan a separarse más de $30 \mathrm{~m}$. Las dataciones han asignado una cronología de $14{ }^{10} \mathrm{Be} \mathrm{ka}$ a la morrena exterior y $12^{10} \mathrm{Be}$ ka a la morrena interior. En el Nevado Illimani, Smith et al., (2011) han descrito morrenas de 12 a $10^{10} \mathrm{Be}$ ka que se superponían o estaban tan sólo a unas decenas de metros de distancia de otras con $15^{10} \mathrm{Be} \mathrm{ka}$.

Es un hecho que a medida que se publican nuevas cronologías glaciales de diferentes regiones de los Andes Centrales su interpretación es más compleja. Frente a la posibilidad de que el LLGM se hubiese adelantado con respecto al LGM global (Smith et al. 2005a), trabajos posteriores han descrito morrenas depositadas por el último máximo avance de las masas de hielo en fechas mucho más recientes, entre 15 y $12 \mathrm{ka}$. La misma realidad aparece en lugares tan distantes como la Cordillera Blanca Glasser et al. (2009), la Cordillera Real (Smith et al. 2011) o el Nevado Coropuna. Podría considerarse que los criterios geomorfológicos contradicen las hipótesis de un avance glaciar cuyas cronologías decrecen con la latitud 
(Zech et al. 2008; Úbeda, 2010), pero en realidad también las sustentan, porque justifican asincronías como la que representa la SED deducida de la muestra NCNE01 $\left(11.0^{36} \mathrm{Cl} \mathrm{ka}\right)$ para una morrena lateral del Valle Santiago, en la vertiente norte del Nevado Coropuna, en un contexto que incluye otras morrenas con cronologías más dilatadas (figura 2). En muchas áreas de la alta montaña andina se ha observado un desajuste entre la interpretación geomorfológica de las morrenas y las SED de los bloques inscritos en los depósitos. Morrenas localizadas en valles colindantes, claramente atribuibles a la máxima expansión de las masas de hielo por su excelente estado de conservación y la ausencia de depósitos anteriores, sugieren dataciones muy dispares, comprendidas en un rango de edades entre $26-22 \mathrm{ka}$ y $12 \mathrm{ka}$. La revisión de las publicaciones científicas sugiere que la misma secuencia de procesos puede haberse repetido en la Cordillera Blanca y la cordillera Huayhuash, al norte de los Andes Centrales de Perú, en la Cordillera Real y la región de Cochabamba, en la cordillera oriental de Bolivia, y en el complejo volcánico Nevado Coropuna, en la cordillera occidental de Perú. Esta claro que independientemente de que los avances de los paleoglaciares se debiesen a una disminución de la temperatura, un incremento de la humedad o una combinación de ambos factores, las diferencias cronológicas no pueden estar controladas exclusivamente por el clima, porque suceden por igual en valles próximos y regiones muy alejadas, donde el gradiente regional de la aridez se traduce en notables diferencias de los registros de precipitación.

Algunas de las morrenas que se conservan en la actualidad pudieron ser depositadas por un último máximo avance o una serie de máximos avances glaciares sucesivos, en los que la expansión final terminó de modelar el conjunto, según el modelo de agregación propuesto por Gibbons et al. (1984). La progresión de las masas de hielo observada en Escandinavia en los años 90' del siglo XX ha quedado registrada por evidencias geomorfológicas muy parecidas. En algunos lugares, dependiendo de cada valle y su contexto geomorfológico, pueden reconocerse hasta seis morrenas o sólo una, resultado de la agregación de todos los depósitos (Winkler y Matthews, 2010). En otros trabajos (Briner et al. 2005; Fabel et al. 2005; Reuther et al.2006) se ha señalado que la agregación de morrenas interfiere en la interpretación de las SED de bloques morrénicos. La mejor solución a ese problema consiste en realizar un análisis geomorfológico suficientemente detallado, delimitando con la máxima precisión posible las formas del relieve. Tanto en el sector NE del Nevado Coropuna como en otras regiones de los Andes Centrales, buena parte de las asincronías glaciales pueden atribuirse a que en unos casos los reavances recientes no alcanzaron las mismas posiciones que en fases anteriores y en otros se quedaron muy próximos o incluso las superaron. Cuando eso sucede los bloques proporcionan una estimación de la edad mínima del grupo de morrenas.

En cualquier caso es evidente que durante el Late Glacial sucedió una fase de expansión glaciar muy importante en los Andes Centrales, que no muestran solo las dataciones de morrenas, sino también otros proxies paleoclimáticos:

1) La estratigrafía de testigos de hielo de glaciares tropicales muestra un claro descenso de la relación isotópica $\delta^{18} \mathrm{O}$ en 14.0-11.5 ka (Thompson et al., 1995, 1998; Ramirez et al., 2003) y la de testigos de sedimentos de lagos del Altiplano importantes cambios durante el mismo periodo (Baker et al., 2001, Rowe et al., 2003 y Placzek et al., 2006), aunque no hay un consenso claro sobre la distribución de los periodos húmedos y secos. 
2) Los registros de SST en las Islas Galápagos (Lea et al., 2006) reflejan una tendencia al calentamiento desde $26-22{ }^{14} \mathrm{C}$ cal ka, coherente con la elevación del nivel medio de los océanos desde $26 \mathrm{ka}$, que sucede a su mínimo valor de los últimos $50 \mathrm{ka}$, (Clark et al., 2009).

3) Sin embargo, el plancton de agua dulce del Lago Titicaca (Fritz et al., 2007), que se extingue en $25{ }^{14} \mathrm{C}$ cal ka, reaparece en $23{ }^{14} \mathrm{C}$ cal ka con una tendencia tan positiva que en $11-9{ }^{14} \mathrm{C}$ cal ka alcanza el nivel más alto de los últimos $40 \mathrm{ka}$.

La revisión de los datos ofrece más evidencias paleoclimáticas. Las tendencias de la SST en las Islas Galápagos y el plancton de agua dulce del Lago Titicaca están interrumpidas por episodios puntuales con máxima depresión de la SST y máxima presencia de plancton de agua dulce, en 22, 20, 17, 13 y $9{ }^{14} \mathrm{C}$ cal ka (figura 5). Esas dataciones de fluctuaciones frías del clima de los Andes Centrales son coherentes con las cronologías de los eventos Heinrich y Younger Dryas, en el Atlántico Norte. La evolución del planctón de agua dulce demuestra

Figura 5

CRONOGRAMA PARA LA CONTEXTUALIZACIÓN PALEOCLIMÁTICA DE LAS SED

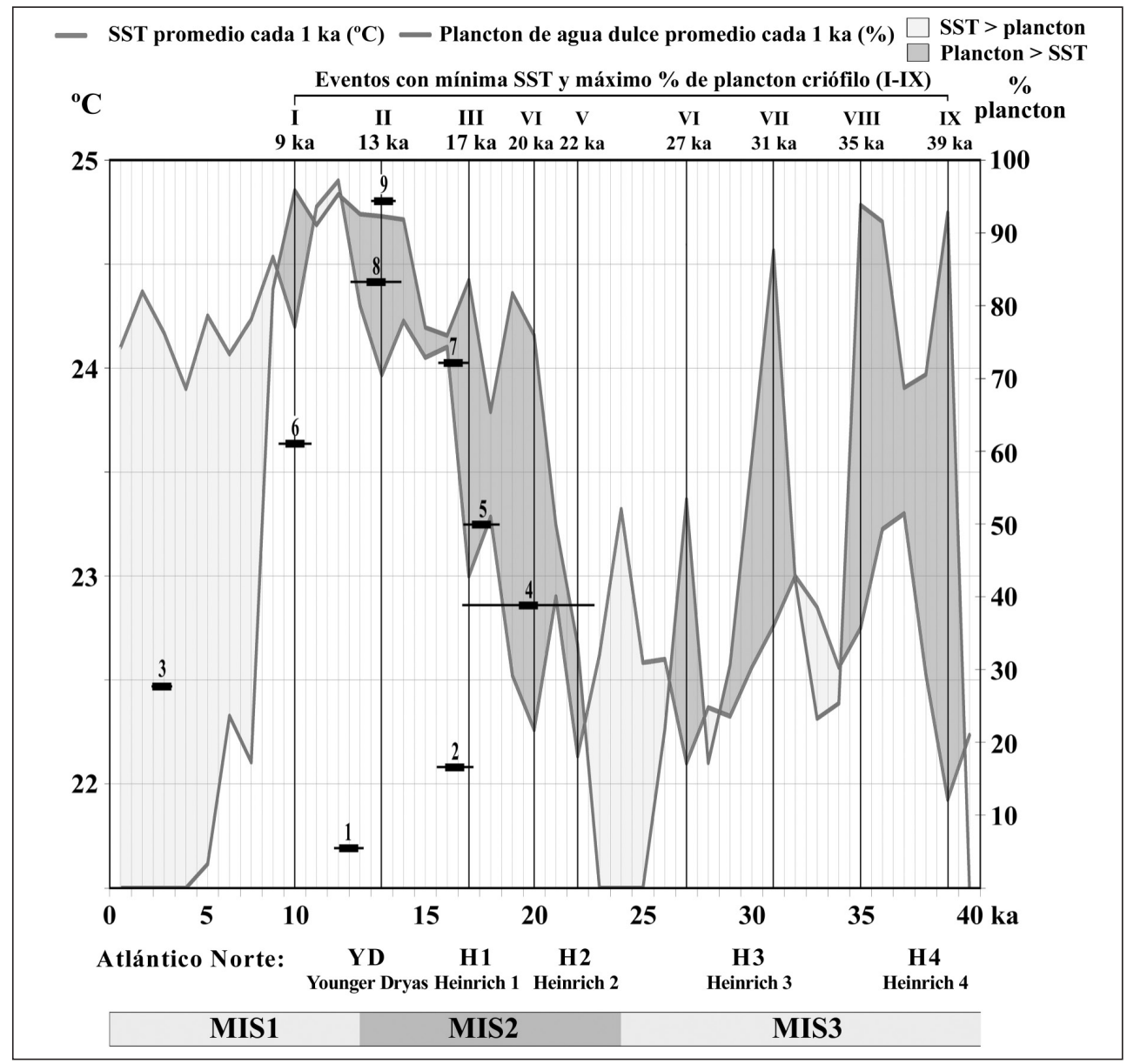


que las condiciones ambientales del altiplano fueron favorables a la expansión glaciar hasta $9^{14} \mathrm{C}$ cal ka, momento en el que la población inicia una drástica caída hasta practicamente extinguirse en $4{ }^{14} \mathrm{C}$ cal ka.

El periodo de la última máxima expansión del sistema glaciar del Nevado Coropuna ( $L L G M-L L G$ ), debió comenzar en $23-22{ }^{14} \mathrm{C}$ cal ka, coincidiendo con el incremento del plancton de agua dulce en el Lago Titicaca y la mayor depresión de la SST en las Islas Galápagos. A continuación, a medida que aumentó la depresión de la paleoELA, las masas de hielo culminaron sucesivamente su progresión en función de la altitud de su área fuente. El final de la glaciación local está representado por las SED deducidas de las muestras NCNE 08 y NCNE 09 , procedentes de las morrenas del valle Jellojello (con un promedio de $12.5 \pm 0.9^{36} \mathrm{Cl} \mathrm{ka}$ ), y la muestra NCNE01 $\left(11.0^{36} \mathrm{Cl} \mathrm{ka}\right)$, que refleja el último reavance en el valle Santiago, donde el altiplano impedía que los paleoglaciares descendiesen a menor altitud y el balance de masa positivo se traducía en una creciente acumulación de hielo. Además de con la evolución del plancton de agua dulce del Lago Titicaca, esa secuencia cronológica también es coherente con las SED deducidas por Zech et al (2007) para los reavances finales en los valles San Francisco, Río Suturi y Huama Loma (entre 13.8 y $11.3^{10} \mathrm{Be} \mathrm{ka}$ ) y Smith et al (2011) al sur del Nevado Illimani, con un promedio de $12.9 \pm 0.5{ }^{10} \mathrm{Be}$ ka.

\section{CONCLUSIONES}

Ocho SED de ${ }^{36} \mathrm{Cl}$ de bloques inscritos en depósitos morrénicos indican que las masas de hielo del sector NE del complejo volcánico Nevado Coropuna experimentaron una última máxima expansión hacia $22 \mathrm{ka}$ antes del presente, seguida por nuevas pulsaciones que se prolongaron hasta $11 \mathrm{ka}$. Esa interpretación es coherente con los resultados obtenidos previamente por otros investigadores en el complejo volcánico, utilizando como cronoindicador otro isótopo cosmogénico $\left({ }^{3} \mathrm{He}\right)$. La evolución de la SST en el archipiélago de las Islas Galápagos muestra un incremento desde $18 \mathrm{ka}$, compatible con el calentamiento global del Holoceno. Sin embargo, el plancton de agua dulce inscrito en los sedimentos del Lago Titicaca sugiere que a pesar de esa tendencia planetaria las condiciones ambientales en los Andes Centrales meridionales continuaron favoreciendo la expansión de las masas de hielo hasta $9 \mathrm{ka}$. Las cronologías glaciales del Nevado Coropuna son compatibles con el contexto ambiental que se deduce de los proxies y el repertorio de dataciones cosmogénicas glaciales de los Andes Centrales. Las cronologías de territorios glaciados muestran considerables diferencias. En algunos casos esas asincronías pueden justificarse con argumentos paleoclimáticos, asumiendo que durante el Pleistoceno final el LLGM avanzó progresivamente hacia el sur y el oeste de la cordillera, a medida que se en las regiones más áridas se elevó la humedad del clima. El incremento de las precipitaciones habría sostenido la expansión de las masas de hielo, enlazando el LLGM con un LLG que se prolongaría hasta bien entrado el Holoceno. Por otra parte, muchas SED glaciales sólo pueden explicarse a través de un análisis geomorfológico detallado, que en esos casos suele reflejar una sucesión de pulsaciones glaciares de intensidad creciente, con deposición de morrenas próximas entre sí y/o desmantelamiento de las formas pre-existentes. La aplicación en nuevos trabajos de las técnicas de investigación que se han ensayado debe contribuir a corroborar las hipótesis planteadas. 


\section{AGRADECIMIENTOS}

Este trabajo se inscribe en las actividades científicas del Grupo de Investigación en Geografía Física de Alta Montaña de la Universidad Complutense de Madrid. Su realización no habría sido posible sin la financiación del proyecto del Plan Nacional de I+D+i del Gobierno de España CGL2009-7334 y la cooperación de los siguientes organismos: Dirección de Geología Ambiental y Riesgo Geológico del Instituto Geológico, Minero y Metalúrgico de Perú (INGEMMET); Unidad de Glaciología y Recursos Hídricos de la Autoridad Nacional del Agua de Perú(UGRH-ANA); Instituto Geofísico del Perú (IGP) y la ONG Guías de Espeleología y Montaña (GEM), con la colaboración de Canal de Isabel II Gestión (España).

\section{BIBLIOGRAFÍA}

BAKER, P.A., RIGSBY, C.A., SELTZER, G.O., FRITZ, S.C., LOWENSTEINK, T.K., BACHER, N.P. VELIZ, C. (2001): «Tropical climate changes at millennial and orbital timescales on the Bolivian Altiplano». Nature, 409: 8

BALCO, G., STONE, J.O., LIFTON, N.A., DUNAI, T.J. «A complete and easily accessible means of calculating surface exposure ages or erosion rates from $10 \mathrm{Be}$ and $26 \mathrm{Al}$ measurements». Quaternary Geochronology, 3: 174-195.

BRINER, J.P., KAUFMAN, D.S., MANLEY, W.F., FINKEL, R.C., CAFEE, M.W. (2007). «Cosmogenic exposure dating of late Pleistocene moraine stabilization in Alaska». Geological Society of America Bulletin, 117, 7-8: 1108-1120.

BROMLEY, R.M., SCHAEFER, J.M., WINCKELER, G., HALL, B.L., TODD, C.E., RADEMAKER, K.M. (2011a): «Late Pleistocene snowline fluctuations at Nevado Coropuna $\left(15^{\circ} \mathrm{S}\right)$, southern Peruvian Andes». Journal of Quaternary Science, 26 (3): 305-317.

BROMLEY, R.M., HALL, B.L., SCHAEFER, J.M., WINCKELER, G., TODD, C.E., RADEMAKER, K.M. (2011b): «Glacier fluctuations in the southern Peruvian Andes during the late-glacial period, constrained with cosmogenic ${ }^{3} \mathrm{He}$. Journal of Quaternary Science, 26 (1): 37-43.

BROMLEY, R.M., HALL, B.L., SCHAEFER, J.M., WINCKELER, G., TODD, C.E., RADEMAKER, K.M. (2009): «Relative timing of last glacial maximum and late-glacial events in the central tropical Andes». Quaternary Science Reviews, 1-13.

CALDAS, J. (1993): Geología de los cuadrángulos de Huambo y Orcopampa. Lima (Perú), Instituto Geológico, Minero y Metalúrgico del Perú (INGEMMET), Lima (Perú).

CLAPPERTON, C. (1993): Quaternary Geology and Geomorphology of South America. Elsevier, Amsterdam (Holanda).

CLARK, P.U., DYKE, A.S., SHAKUN, J.D., CARLSON, A.E., WOHLFARTH, B., MITROVICA, J.X., HOSTETLER, S.W., MCCABE, A.M. (2009). «The Last Glacial Maximum». Science, 325: 710-714.

DORNBUSCH, U. (2002). «Pleistocene and present day snowlines rise in the Cordillera Ampato, Western Cordillera, southern Peru». Neues Jahrbuch für Geologie und Paläeontologie Abhandlungen, 225: 103-126.

FRITZ, S.C., BAKER, P.A., SELTZER, G.O., BALLANTYNE, A., TAPIA, P., CHENG, H., EDWARDS, R.L. (2007): Lake Titicaca 370KYr LT01-2B Sediment Database, Lake Titi- 
caca 370KYr LT01-2B Sediment Data. IGBP PAGES/World Data Center-A for Paleoclimatology Data Contribution Series \# 92-008. NOAA/NGDC Paleoclimatology Program. Boulder (EEUU).

GIBBONS, A., MEGEATH, J., PIERCE, K. (1984): «Probability of moraine survival in a succession of glacial advances». Geology, 12(6): 327-30.

GLASSER, N., CLEMMENS, S., SCHNABEL, C., FENTON, C.R., MCHARQUE, L. (2009): «Tropical glacier fluctuations in the Tropical Cordillera Blanca, Perú». Artic, Antartic and Alpine Research, 36: 100-107.

HALL, S., FARBER, D. L., RODBELL, D.T., SMITH, J.A., MARK, B.G., KASSEL, C. (2009): «Geochronology of Quaternary glaciations from the tropical Cordillera Huayhuash, Peru». Quaternary Science Review,. 28: 2931-3009.

IPCC, (2007): Contribution of Working Groups I, II and III to the Fourth Assessment Report of the Intergovernmental Panel on Climate Change. In: R.K. Pachauri and A. Reisinger (Editores), Geneva, Switzerland, pp. 104.

KASER, G., OSMASTON, H. (2002): Tropical Glaciers. Cambridge University Press. Cambridge (Reino Unido).

KERSCHNER, H., IVY-OCHS, S. (2008): «Palaeoclimate from glaciers: Examples from the Eastern Alps during the Alpine Lateglacial and early Holocene». Global and Planetary Change, 60: 58-71.

KLEIN, A.G., SELTZER, G.O., ISACKS, B.L. (1999): «Modern and Last Local Glacial Maximum snowlines in the Central Andes of Peru, Bolivia, and Northern Chile». Quaternary Research Reviews, 18: 3-84.

KULL, C., GROSJEAN, M. (2000): «Late Pleistocene climate conditions in the North Chilean Andes drawn from a climate-glacier model». Journal of Glaciology, 46: 622-632.

LAL, D. (1991): «Cosmic ray labeling of erosion surfaces: in situ nuclide production rates and erosion models». Earth and Planetary Science Letters, 104: 424-439.

LEA, D.W., PAK, D.K., BELANGER, C.L., SPERO, H.J., HALL. M.A., SHACKELTON, N. J. (2006): Galápagos TR163-22 Foraminiferal $d^{18} O$ and $M g / C a$ Data and SST Reconstruction. IGBP PAGES/World Data Center for Paleoclimatology Data Contribution Series \# 2006-090. NOAA/NCDC Paleoclimatology Program, Boulder (EEUU).

LICCIARDI, J.M., SCHAEFER, J.M., TAGGART, J.R., LUND, C.D. (2009). «Holocene glacier fluctuations in the Peruvian Andes indicate northern climate linkages». Science, 325: 1677-1679.

LIFTON, N.A., BIEBER, J.W., CLEM, J.M., DULDIG, M.L., EVENSOM, P., HUMBLE, J. E., PYLE, R. (2005: «Addressing solar modulation and long-term uncertainties in scaling secondary cosmic rays for in situ cosmogenic nuclide applications». Earth and Planetary Science Letters, 239: 140-161.

MARK, B.G., HARRISON, S.P., SPESSA,A., NEWE, M., EVANS, D.J.A., HELMENSG, K.F. (2005). «Tropical snowline changes at the last glacial maximum: A global assessment.» Quaternary International 138-139: 168-201.

MATTHEWS, J.A., WINKLER (2011): «Schmidt-hammer exposure-age dating (SHD): application to early Holocene moraines and a reappraisal of the reliability of terrestrial cosmogenic-nuclide dating (TCND) at Austanbotnbreen, Jotunheimen, Norway». Boreas, 40: $256-270$. 
NESJE, A., DAHL, S.O. (2000): Glaciers and Environmental Change. Arnold, Londres (Reino Unido).

NISHIIZUMI, K., LAL, D., KLEIN, J., MIDDLETON, R., ARNOLD, J.R. (1989): «Cosmic ray production rates of ${ }^{26} \mathrm{Al}$ and ${ }^{10} \mathrm{Be}$ in quartz from glacially polished rocks». Journal of Geophysical Research, 94: 17907-17915.

NÚÑEZ, S., VALENZUELA, G. (2001): Mapa Preliminar de Amenaza Volcánica Potencial del Nevado Coropuna. Instituto Geológico, Minero y Metalúrgico del Perú (INGEMMET). Lima (Perú).

OLSCHAUSKY, E., DÁVILA, D. (1994): Geología de los cuadrángulos de Chuquibamba y Cotahuasi. Instituto Geológico, Minero y Metalúrgico del Perú (INGEMMET). Lima (Perú).

PHILLIPS, F.M. (2003): «Cosmogenic ${ }^{36} \mathrm{Cl}$ ages of Quaternary basalt flows in the Mojave Desert, California, USA». Geomorphology, 53: 199-208.

PLAZCEK, C. (2005): Stratigraphy, geochronology, and geochemistry of paleolakes on the southern bolivian altiplano. (Tesis Doctoral). Department of Geosciences. Universidad de Arizona, Tucson (EEUU). 252.

PLAZCEK, C., QUADE, J. PATCHETT, P.J. (2006): «Geochronology and stratigraphy of late Pleistocene lake cycles on the southern Bolivian Altiplano». Geological Society of America Bulletin, 118: 515-532.

RACOVITEANU, A. (2004): Sacred Mountauins and Glacial Archeology in the The Andes. Department of Geography. University of Colorado (EEUU): 167.

RACOVITEANU, A. MANLEY, W.F., ARNAUD, Y., MARK, W.W. (2007): «Evaluating Digital Elevation Models for Glaciologic Applications. An example from Nevado Coropuna, Peruvian Andes». Global and Planetary Change, 59: 110-125.

RAMÍREZ, E., HOFFMANN, G., TAUPIN, J.D., FRANCOU, B., RIBSTEIN, P., CAILLON, N., FERRON, F.A., LANDAIS, A., PETIT, J.R., POUYAUD, B., SCHOTTERER, U., SIMOES, J.C., STIEVENARD, M. (2003) : «A new Andean deep ice core from Nevado Illimani (6350 m), Bolivia». Earth and Planetary Science Letters, 212: 337-350.

REUTHER, A.U., HERGET, J., IVY-OCHS, S., BORODAVKO, P., KUBIK, P.W., HEINE, K. (2006): «Constraining the timing of the most recent cataclysmic flood event from icedammed lakes in the Russian Altai Mountains, Siberia, using cosmogenic in situ ${ }^{10} \mathrm{Be} »$. Geology, 34: 913-916.

ROWEA, H.D., GUILDERSON, T.P., DUNBAR, R.B., SOUTHONC, J.R., SELTZER, G.O., MUCCIARONE, D.A., FRITZ, S.C., BAKER, P.A. (2003): «Late Quaternary lakelevel changes constrained by radiocarbon and stable isotope studies on sediment cores from Lake Titicaca, South America». Global and Planetary Change, 38: 273-290.

SELTZER, G.O. (1990): «Recent glacial history and paleoclimate of the Peruvian-Bolivian Andes». Quaternary Science Reviews, 9: 137-152.

SMITH, C.A., LOWEL, T.V., OWEN, L.A., CAFFEE, M.W. (2011): «Late Quaternary glacial chronology on Nevado Illimani, Bolivia, and the implications for paleoclimatic reconstructions across the Andes», Quaternary Research, 75: 1-10.

SMITH, J.A., FINKEL, R., FARBER, D.A., RODBELL, D.T., SELTZER, G.O. (2005): «Moraine preservation and boulder erosion in the tropical Andes: interpreting old surface exposure ages in glaciated valleys». Journal of Quaternary Science, 20 (7-8): 735-758. 
SMITH, J.A., SELTZER, G.O., RODBELL, D.T., KLEIN, A.G. (2005): «Regional synthesis of last glacial maximum snowlines in the tropical Andes, South America». Quaternary International, 138-139: 145-167.

SMITH, J.A. (2005): Timing and extent of glaciation in the tropical Andes. Tesis Doctoral. Syracuse (USA), Syracuse University: 173.

SMITH, J.A., MARK, B.G., RODBELL, D.T. (2008): «The timing and magnitude of mountain glaciation in the tropical Andes». Joumal of Quaternary Science, 23: 609-634.

STONE, J.O. (2000): «Air pressure and cosmogenic isotope production». Journal of Geophysical Research, 105: 23753-23759.

THOMPSOM, L.G., MOSLEY-THOMPSON, E., BOLZAN, J. F., KOCI, B. R. (1995): «Late glacial stage and Holocene tropical ice core records from Huascarán, Peru». Science, 269: 46-50.

THOMPSOM, L.G., DAVIS, M., MOSLEY-THOMPSON, E., SOWERS, T.A., HENDERSON, K.A., ZAGORODNOV, V.S., LIN, P.N., CAMPEN, R.K., BOLZAN, J.F., COLEDAI, J. ,FRANCOU, B. (1998): «A 25,000 year tropical climate history from Bolivian ice cores». Science, 282: 1858-1864.

ÚBEDA, J. (2011): El impacto del cambio climático en los glaciares del complejo volcánico Nevado Coropuna (cordillera Occidental de los Andes, Sur del Perú)» Tesis Doctoral. Departamento de Análisis Geográfico Regional y Geografía Física. Universidad Complutense de Madrid, Madrid (España). 558 pp. Disponible online en el enlace: http://eprints. ucm.es/12076/

ZECH, R., KULL, C., KUBIK, P.W., VEIT, H. (2007): «LGM and Late Glacial glacier advances in the Cordillera Real and Cochabamba (Bolivia) deduced from ${ }^{10} \mathrm{Be}$ surface exposure dating.» Climate of the Past Discussions 3: 839-869.

ZECH, R., MAY, J.H., KULL, C., ILGNER, J., KUBIK, P.W., VEIT, H. (2008): «Timing of the late Quaternary glaciation in the Andes from $\sim 15^{\circ}$ to $40^{\circ} \mathrm{S} »$. Journal of Quaternary Science, 23 (6-7): 635-647.

ZREDA, M.G., PHILLIPS, F.M. (2000): «Cosmogenic Nuclide Buildup in Surficial Materials. Quaternary Geochronology, Methods and Applications». En STRATON NOLLER, SOWERS, J.M., LETTIS, W.R. (Eds.). Americal Geophysical Union: 61-76. Washington (EEUU).

ZREDA, M., ENGLAND, J., PHILliPS, F.M., ELMORE, D., SHARMA, P. (1999): «Unblocking of the Nares Strait by Greenland and Ellesmere ice-sheet retreat 10,000 years ago». Nature, 398: 139-142. 